\title{
Surface effect investigation for static bending of nanowires resting on elastic substrate using Timoshenko beam theory in tandem with the Laplace-Young equation
}

\author{
Amin Khajeansari ${ }^{\mathrm{a}}$ And Gholam Hosein Baradaran \\ Department of Mechanical Engineering, Faculty of Engineering, Shahid Bahonnar, University of Kerman, Kerman 76188, Iran
}

Received 25 Septembre 2011, Accepted 16 April 2012

\begin{abstract}
In the present study, an enriched continuum mechanics framework is employed to study the surface effects on bending behavior of silver nanowires (NWs) resting on elastic substrate. The Timoshenko beam theory and the Laplace-Young equation are employed to investigate static behavior of silver NWs lying on Winkler-Pasternak elastic substrate. Three types of boundary conditions are considered as doubly simply supported (S-S), doubly clamped (C-C) and cantilevered (C-F). Analytical solutions are obtained for NWs with surface crystallographic orientation of [001] subjected to a concentrated external force. By defining different normalized contact stiffness, extensive numerical results are carried out to study the influence of effective parameters such as substrate, surface, aspect ratio $(L / D)$ and diameter on the stiffness of NWs. According to the obtained results, the effect of surface and its rate of variation on stiffness of NWs lying on Winkler and Winkler-Pasternak elastic foundation models are more significant in (C-F) type of boundary condition compared to the NWs without foundation. By increasing the modulus of elastic substrate, the effect of shear deformation increases which it is more considerable in (C-C) and (S-S) NWs resting on the Winkler-Pasternak and Winkler substrate models, respectively.
\end{abstract}

Key words: Nanowire / elastic substrate / surface effect / size dependency / timoshenko beam

\section{Introduction}

Because of the novel physical properties of NWs, many researchers have tried to investigate the superior mechanical properties of these one dimensional nanostructures [1-7]. The extraordinary physical properties of NWs are mainly due to their large ratio of surface per volume and quantum confinement effects. Quantum confinement tightly affects on the optical and electrical properties of the materials. Typically in nanoscale, this phenomenon can also change the mechanical properties of materials by increasing the difference of energy level and band gap in nanomaterial comparing to its bulk state, while in the bulk sample the band gap remains constant at its original energy due to a continuous energy state [8,9]. The excess energy in a nanoscale material respect to corresponding bulk material is related to the particles near the surface of confined dimension. Therefore, the quantum confinement dominates the other processes leading to change the potential energy, force field and mechanical

\footnotetext{
${ }^{a}$ Corresponding author:

amin.khajeansari@eng.uk.ac.ir
}

properties of nanomaterial respect to the bulk phase. As a result, the balance of the surface particles is destroyed and the surface tension changes tremendously and the specific tension and potential energy for the surface are defined [10]. Because of their exceptional physical properties, NWs have found several applications in nanoelectromechanical systems (NEMS). Interesting properties of the NEMS devices typically arise from the behavior of the active parts, which in most cases, are in the forms of cantilevers or doubly clamped beams with dimensions at nanometer scale [11-13].

In recent years, many methods and approaches have been implemented for studying the physical properties of nanostructures such as nanowires, nanotubes, nanoplates, nanocomposites, etc. Atomistic simulations play an important role to detect unexpected properties of NWs at small thickness $[14,15]$. The thickness denotes the diameter of a circular cross section NW. In the case of square cross section, the thickness denotes the length of cross section. For the NWs including a large cluster of atoms with the length scales above several ten of nanometers, the atomistic simulations are too expensive. For investigation the mechanical properties of NWs by 
experimental methods, the conventional tensile and creep testing method have been prohibited, because these testing methods require the size of the sample be sufficiently large to be clamped rigidly by the sample holder without sliding [16]. Therefore, practically the bending deformation experiments have been usually utilized to examine the mechanical properties of NWs. However, the bending experiments have also their individual complexities. Jing et al. [17] and Chen et al. [18] measured the importance of the thickness and boundary conditions on the elastic properties of silver NWs respectively. To explain the size related elastic properties of $\mathrm{ZnO}$ NWs in their experiments, Chen et al. [19] proposed a core-shell model, where the nanowire is treated as a composite with a shell and a core structure.

Enriched continuum mechanics models have been established to simplify the analysis of nanosystems. A simplified model for prediction of the effective elastic properties of nanosized structural elements based on the standard continuum mechanics and atomistic simulation results is demonstrated in [20]. A theoretical model is presented in [21] to investigate the size dependent bending behavior of NWs with taking into account the influence of both the atomic configuration at the relaxed surface and the surface tension. Li et al. [22] proposed a continuum approach based on the strain gradient theory for determination of the size effects in NWs under bending deformation. Among related studies, a valuable enriched continuum mechanics model proposed by Gurtin and Murdoch [23]. They introduced a non-classical phenomenon known as the surface/interface effect which is taken into account for prediction of the size dependent elastic properties of nanomaterials. This model is prepared by embedding the surface energy into the conventional continuum mechanics model. In this continuum model, the main approach is that the nanoscale body is considered as a bulk volume surrounded by a surface layer. Dingreville et al. [24] proposed a methodology to incorporate the surface energy into the continuum theory to model the size-dependent effective moduli of isolated nanosized elements (particles, wires and films). The closed form relations have been proposed by Duan et al. [25-27] to characterize the effective properties of nanocomposites containing interface stress. Sharma et al. [28, 29] have studied the sizedependent elastic state of non-homogenous nanomaterials containing inside inclusions. The numerical approaches such as FEM and XFEM from the continuous models of nanowires and nonocomposites were proposed by Yvonnet et al. $[30,31]$ to simulate the surface/interface effects. In their approaches, the surface elastic parameters were computed from atomistic simulations.

Recently an enriched continuum mechanics approach has been presented by Wang and Feng [32-35] to investigate the size dependent mechanical properties of NWs by taking into account both the surface elasticity and the surface stress. They evaluated the effect of surface on contact, free vibration and axial buckling behavior of NWs under static loads using the Euler-Bernoulli and the Timoshenko beam theories and linear elasticity.
Comparing variation of the Young modulus versus diameter of NWs with the associated experimental results in He and Lilley [36] and Jiang and Yan [37], confirms the applicability of the above method to predict the size dependency of mechanical properties of NWs. In novel technologies or materials the NWs implement as key components in flexible electronics, nanocircuitry, nanosensors, nanoprocessors, electronic logic gates, renewable energy technologies and biological or gas sensing applications [1$3,5-7,11-13]$. Thereby the NWs can be deposited on a substrate. In NEMS, are mentioned nanodevices at above and/or other similar future application [38,39]. Therefore surveying the mechanical behavior of NWs resting on the substrate medium can be considerable. In recent years, many researchers have studied the mechanical behavior of nanoscale materials especially carbon nanotubes and graphene sheets embedded in various surroundings $[40-42]$.

To the best of our knowledge, effect of surface on static bending of NWs on elastic substrate by considering the shear deformation effect has not investigated yet. In the present paper, an enriched continuum mechanics model is employed to investigate the effects of residual surface stress and surface elastic constants on the bending deformation of NWs lying on elasticsubstrate. The linear surface elasticity theory combined with the Laplace-Young equation to model the surface effects and Winkler and Pasternak elastic foundations model is also applied to simulate the substrate medium. The shear deformation effect is considered by assuming Timoshenko beam theory and an explicit solution is obtained for transverse displacement and stiffness of NWs with three different loads and boundary conditions: (a) cantilever (C-F) with a concentrated force at the free end, (b) simply supported (S-S) and $(\mathrm{c})$ clamped $(\mathrm{C}-\mathrm{C})$ under concentrated forces at the middle point.

\section{Formulation of the problem}

The Winkler elastic foundation model for a beam is recognized as groups of closely spaced vertical linear elastic springs lying under the beam surface. The foundation modulus is determined by the stiffness of the springs and the reaction force of foundation taken to be linearly proportional to the beam deflection at any point $[43,44]$. The Winkler model does not take into account the continuity and cohesion of the elastic environment. A more realistic and accurate model of the elastic foundation can be obtained by a two parameters elastic foundation which is known as the Winkler-Pasternak model. The Pasternak elastic foundation model [45] simulate the transverse shear stress due to the shear deformation of the medium, while the Winkler foundation model account for the normal pressure from the surrounding elastic medium. In Figure 1, a part of deformed NW with length under bending deformation and external static transverse load $q(x)$ is shown. The NW is modeled with a Timoshenko beam element lying on Winkler-Pasternak elastic substrate model. 


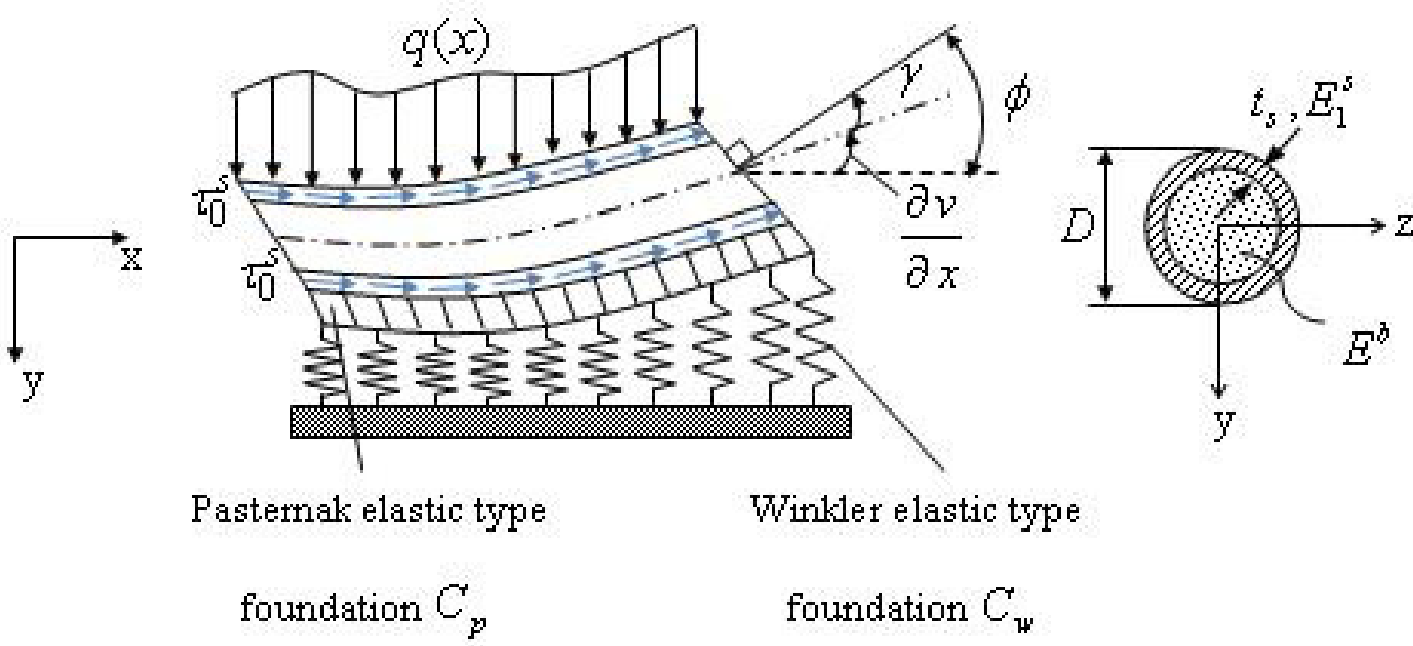

Fig. 1. (a) Shear deformation effect on the configuration of an element of NW on Winkler-Pasternak elastic type foundation, (b) the circular cross section of NW including different elastic modulus of bulk and surface layer with the assumption of $t \ll D$.

The model contains the outer surface layer with the residual surface stress which is represented by the blue arrows in longitudinal direction of NW and the bulk volume at the core.

The surface stress $\boldsymbol{\tau}^{s}$ is related to the surface strain $\varepsilon^{s}$ through the Shuttleworth's equation [46],

$$
\boldsymbol{\tau}^{s}=\tau_{0}^{s} \boldsymbol{I}+\frac{\partial \gamma\left(\varepsilon^{s}\right)}{\partial \varepsilon^{s}}
$$

where $\gamma$ is the surface strain energy density and $\boldsymbol{\tau}_{0}^{s}$ is the strain-independent surface/interfacial stress at $\varepsilon^{s}$, which is known as residual surface stress and $\boldsymbol{I}$ is the unit tensor for the surface. The surface elasticity tensor $\underline{\underline{S}}$ is defined by

$$
\tau^{s}=\tau_{0}^{s} \boldsymbol{I}+\underline{\underline{S}}: \varepsilon^{s}
$$

where $\underline{\underline{S}}$ is specified as

$$
\underline{\underline{\boldsymbol{S}}}=\left.\frac{\partial \boldsymbol{\tau}^{s}\left(\varepsilon^{s}\right)}{\partial \varepsilon^{s}}\right|_{\varepsilon^{s}=0}
$$

In fact the positive definiteness of the bulk elastic modulus tensor which satisfies the stability of the solid cannot be applied to the surface elastic tensor. This is arising from the fact that the surface cannot exist independent of the bulk, and the total energy which is compound of the bulk energy and the surface energy, needs to satisfy the positive definiteness condition of the elastic modulus tensor of the whole material [10].

In one dimensional bending problems presented in this study, the relation between surface stress and infinitesimal surface strain in longitudinal direction is reduced to

$$
\tau^{s}=\tau_{0}^{s}+E^{s} \varepsilon^{s}
$$

where $E^{s}\left(\mathrm{~N} . \mathrm{m}^{-1}\right)$ is the surface elastic modulus which is calculated from the surface elastic constants [36]:

$$
E^{s}=\frac{\left(S_{1111}+2 S_{1122}\right)\left(S_{1111}-S_{1122}\right)}{\left(S_{1111}+S_{1122}\right)}
$$

According to Figure 1b the circular cross section of the NW is a composite material compound of a bulk volume with the elastic modulus of $E^{b}$, the thickness of $D$ and a surface layer around the cross section by the elastic modulus and thickness of $E_{1}^{s}$ and $t^{s}$ respectively. The thickness of the surface layer $t^{s}$ is assumed to be much smaller than the thickness of the cross section $t^{s} \ll D$. The relationship between $E_{1}^{s}$ and $E^{s}$ is stated as $E^{s}=E_{1}^{s} t^{s}$ [32].

Based on the composite beam theory and with the assumption that $t^{s} \ll D$, the effect of surface elasticity is taken into account in the effective flexural rigidity of NW as follows [36].

$$
(E I)^{*}=E^{b} I^{b}+E_{1}^{s} I^{s}=\frac{\pi E^{b} D^{4}}{64}+\frac{\pi}{8} E^{s} D^{3}
$$

Due to the presence of surface stress, the traction vector field across the outer surface of the NW is discontinuous and has to verify the Laplace-Young equation as follows [33-36]

$$
\left\langle\sigma_{i j}^{+}-\sigma_{i j}^{-}\right\rangle n_{i} n_{j}=\tau_{\alpha \beta}^{s} \kappa_{\alpha \beta}
$$

$\left\langle\sigma_{i j}^{+}-\sigma_{i j}^{-}\right\rangle$is the stress jump across the surface, $\sigma_{i j}^{+}$and $\sigma_{i j}^{-}$represent the stress at upmost and bottommost layer of the surface respectively. $n_{i}$ is the unit vector normal to the surface, $\tau_{\alpha \beta}^{s}$ is the surface stress and $\kappa_{\alpha \beta}$ denotes the curvature tensor. Applying the Timoshenko beam theory and assuming small deformation, the curvature $\kappa$ is approximated by the first derivative of the rotation angle of the beam cross section due to pure bending as $\varphi^{\prime}(x)$.

The stress tensor $\sigma_{i j}$ is generally acting on the volume of the body and is three dimensional. Therefore the indices of $i, j$ varies as $i, j=1,2,3$ while the surface stress acting in a two dimensional surface intrinsically and the Greek indices take the values $\alpha, \beta=1,2$. Assuming small deflection of the beam, a distributed transverse load $q_{s}(x)$ is imposed along the longitudinal direction of deformed 
NW which is coming from the stress jump described in Equation (7).

$$
q_{s}(x)=H \varphi^{\prime}(x)
$$

The parameter $H$ for circular cross section is defined by $[36]$.

$$
H=2 \tau_{0}^{s} D
$$

The direction of $q_{s}(x)$ depends on the signs of deformed beam curvature and residual surface stress of the beam. It is noted that the sign of the residual surface stress of materials depends on the surface crystallographic direction and can be either positive or negative. In Equations (6) and (8), are captured the surface parameters effects as the surface Young modulus $E^{s}$ and the residual surface stress $\tau_{0}^{s}$ in analysis of the NW.

By this approach, determining the mechanical properties and behaviors of the different nanomaterials with different surface crystallographic orientations is straightforward. Basically, it is one of the important advantages of the above approach.

In static bending deformation of beams, when dimensions of the cross-section are not small compared to the length, it is needed to consider the effect of shear deformation. This effect is modeled by Timoshenko beam theory which is well-known as the thick beam theory. As it is seen in Figure 1

$$
\gamma=\varphi-\frac{\partial v}{\partial x}
$$

where the parameter of $\gamma$ signifies the shear deformation and $v$ is the transverse displacement of the beam. The bending moment $M$ and shear force $V$ are functions of $v$ and $\varphi$ given as follows

$M(x)=-(E I)^{*} \frac{\partial \varphi(x)}{\partial x}, \quad V(x)=\alpha_{s} A G\left(\frac{\partial v(x)}{\partial x}-\varphi(x)\right)$

where $G$ is the shear modulus, $A$ is the cross-section of area and $\alpha_{s}$ is the shear coefficient which is function of the cross sectional shape. The shear coefficient is $\alpha_{s}=5(1+v)(6+5 v)^{-1}$ for a rectangular cross section, and is $\alpha_{s}=6(1+v)^{2}\left(7+12 v+4 v^{2}\right)^{-1}$ for a circular cross section [47]. Based on the Timoshenko beam theory, the governing differential equations for static bending deformation of a NW under the total transverse distributed force $p(x)$ are given by:

$$
\begin{aligned}
& (E I)^{*} \frac{\partial^{2} \varphi}{\partial x^{2}}-\alpha_{s} A G \varphi+\alpha_{s} A G \frac{\partial v}{\partial x}=0 \\
& \alpha_{s} A G \frac{\partial^{2} v}{\partial x^{2}}-\alpha_{s} A G \frac{\partial \varphi}{\partial x}=-p(x)
\end{aligned}
$$

In presence of surface effect and Winkler-Pasternak elastic type substrate, the total transverse distributed force $p(x)$ acting on $\mathrm{NW}$ is as follows

$$
p(x)=q_{s}(x)+q_{f}(x)+q(x)
$$

where $q_{s}(x), q_{f}(x)$ and $q(x)$ are the transverse distributed forces corresponding to residual surface stress and two parameters elastic foundation and external stimulus respectively. $q_{f}(x)$ can be written as [48]

$$
q_{f}(x)=-C_{w} v(x)+C_{p} \nabla^{2} v(x)
$$

The Winkler modulus $C_{w}\left(\mathrm{nN}^{\mathrm{nnm}}{ }^{-2}\right)$ and Pasternak modulus $C_{p}(\mathrm{nN})$ are computed by multiplying the diameter of the NW by the Winkler and Pasternak stiffness constants of $K_{w}\left(\mathrm{nN} . \mathrm{nm}^{-3}\right), K_{p} \quad\left(\mathrm{nN} \cdot \mathrm{nm}^{-1}\right)$ respectively. This is needed for the consistency of term units of Equation (14) that they should be nN.nm ${ }^{-1}$.

By substituting Equations (8) and (15) into Equation (14), $p(x)$ is determined by

$$
p(x)=H \varphi^{\prime}(x)-C_{w} v(x)+C_{p} \nabla^{2} v(x)+q(x)
$$

It must be mentioned that in the present study $q(x)$ is zero because the external load is imposed to the NW as a concentrated force, so, the effect of concentrated external force is accounted in the boundary conditions. The substrate medium of NWs is assumed to be made up from polymer. According to the values of Winkler and Pasternak stiffness utilized by Liew [49], the nondimensional modulus parameters are defined as [50]

$$
\hat{C}_{w}=\frac{C_{w} L^{4}}{(E I)^{*}}, \quad \hat{C}_{p}=\frac{C_{p} L^{2}}{(E I)^{*}}
$$

where $\hat{C}_{w}$ and $\hat{C}_{p}$ represent the non-dimensional Winkler and Pasternak modulus parameters respectively.

In order to decouple Equations (12) and (13), we use Equation (13) and its second derivative to determine $\varphi^{\prime}$ and $\varphi^{\prime \prime \prime}$ in terms of derivatives of $v$; then with substituting $\varphi^{\prime}$ and $\varphi^{\prime \prime \prime}$ in the first derivative of Equation (12), the following differential equation is derived:

$$
\begin{array}{r}
{\left[(E I)^{*}\left(\alpha_{s} A G+C_{p}\right) \frac{\mathrm{d}^{4} v(x)}{\mathrm{d} x^{4}}-\left[(E I)^{*} C_{w}+\left(\alpha_{s} A G H+C_{p}\right)\right]\right]} \\
\times \frac{\mathrm{d}^{2} v(x)}{\mathrm{d} x^{2}}+\left[\alpha_{s} A G C_{w}\right] v(x)=0
\end{array}
$$

The general solution for Equation (16) can be written as

$$
v(x)=A e^{m x}
$$

Substituting Equation (19) into Equation (18) yield the following characteristic equation

$$
\begin{aligned}
{\left[(E I)^{*}\left(\alpha_{s} A G+C_{p}\right)\right] m^{4} } & -\left[(E I)^{*} C_{w}+\alpha_{s} A G\left(H+C_{p}\right)\right] \\
& \times m^{2}+\left[\alpha_{s} A G C_{w}\right] m=0 \quad(20)
\end{aligned}
$$

The roots of above equation can be obtained as

$$
\begin{gathered}
m_{1,2}= \pm \xi_{1} \\
m_{3,4}= \pm \xi_{2} \\
\xi_{1}=\frac{\sqrt{2}}{2 \alpha} \sqrt{\alpha[\beta+\sqrt{\lambda}]} \\
\xi_{1}=\frac{\sqrt{2}}{2 \alpha} \sqrt{\alpha[\beta-\sqrt{\lambda}]}
\end{gathered}
$$

where

$$
\begin{aligned}
\alpha & =(E I)^{*}\left(\alpha_{s} A G+C_{p}\right) \\
\beta & =(E I)^{*} C_{w}+\alpha_{s} A G\left(H+C_{p}\right) \\
\lambda & =\beta^{2}-4 \alpha\left(\alpha_{s} A G C_{w}\right)
\end{aligned}
$$


In the present study, the substrate medium assumed to be polymer and the range of non-dimensional Winkler and Pasternak modulus parameter values are considered as the same as those used by Liew et al. [49]. Consequently, two circumstances may occur in the solution process according to the roots of characteristic Equation (20). The values of $\xi_{1}$ and $\xi_{2}$ are complex conjugate if $\lambda<0$, and they are real if $\lambda>0$. When the roots of characteristic Equation (20) are complex conjugate as $m_{1,2,3,4}= \pm p \pm i q$, the general solution for the governing equation of a NW lying on two parameters elastic type substrate medium based on the Timoshenko beam theory is obtained as

$$
\begin{aligned}
v(x)=e^{p x}\left[C_{1} \cos (q x)+\right. & \left.C_{2} \sin (q x)\right]+e^{-p x} \\
& \times\left[C_{3} \cos (q x)+C_{4} \sin (q x)\right]
\end{aligned}
$$

when the roots of the characteristic equation $\left(\xi_{1}\right.$ and $\left.\xi_{2}\right)$ are real, the general solution of the governing Equation (18) becomes,

$$
v(x)=C_{1} e^{\xi_{1} x}+C_{2} e^{\xi_{2} x}+C_{3} e^{\xi_{3} x}+C_{4} e^{\xi_{4} x}
$$

$v(x)$ is the transverse displacement of NW. Determining $\varphi^{\prime \prime}$ from the first derivative of Equation (13) and substituting in Equation (12), $\varphi(x)$ can be written in terms of $v(x)$ as follows

$$
\varphi(x)=\left(\frac{1}{\eta}\right)\left[\alpha \frac{\mathrm{d}^{3} v(x)}{\mathrm{d} x^{3}}+\mu \frac{\mathrm{d} v(x)}{\mathrm{d} x}\right]
$$

where

$$
\begin{aligned}
\eta & =\alpha_{s} A G\left(\alpha_{s} A G-H\right) \\
\mu & =\eta-(E I)^{*} C_{w}
\end{aligned}
$$

when the roots of the characteristic equation to be complex conjugate, the parameters $p$ and $q$ in Equation (23) can be obtained from a mathematical procedure as below

$$
\begin{gathered}
z_{1}=\alpha \beta ; \quad z_{2}=\alpha \sqrt{-\lambda} \\
\theta=\tan ^{-1}\left(\frac{z_{2}}{z_{1}}\right)=\tan ^{-1}\left(\frac{\sqrt{-\lambda}}{\beta}\right) \\
z=\sqrt{z_{1}^{2}+z_{2}^{2}}=\alpha \sqrt{\beta^{2}+\lambda} \\
p=\frac{\sqrt{2}}{2 \alpha}(z)^{1 / 2} \cos \left(\frac{1}{2} \theta\right)=\frac{\sqrt{2}}{2 \alpha}\left(\alpha \sqrt{\beta^{2}+\lambda}\right)^{1 / 2} \\
\times \cos \left(\frac{1}{2} \tan ^{-1}\left(\frac{\sqrt{-\lambda}}{\beta}\right)\right) \\
\left.q=\frac{\sqrt{2}}{2 \alpha}(z)^{1 / 2} \sin ^{1 /} \theta\right)=\frac{\sqrt{2}}{2 \alpha}\left(\alpha \sqrt{\beta^{2}+\lambda}\right)^{1 / 2} \\
\times \sin \left(\frac{1}{2} \tan ^{-1}\left(\frac{\sqrt{-\lambda}}{\beta}\right)\right)
\end{gathered}
$$

In Equations (23) and (24), $C_{1}-C_{4}$ are the unknown coefficients that will be determined from the boundary conditions. In the present study the unknown coefficients have been determined for doubly simply supported (S$\mathrm{S})$, doubly clamped (C-C) and cantilevered (C-F) NWs explicitly. In appendices $\mathrm{A}$ and $\mathrm{B}$ the coefficients $C_{1}-C_{4}$ are given for (S-S) NWs.

The boundary conditions for NWs with two ends simply supported (S-S) and subjected to a concentrated force at the midpoint of the NW are given as

$$
\begin{aligned}
v(0)= & 0 \\
\varphi(L / 2)= & 0 \\
M(0)= & -(E I)^{*} \varphi^{\prime}(0)=0 \\
V(0)= & \alpha_{s} A G\left(v^{\prime}(0)-\varphi(0)\right)-\frac{F}{2}+C_{w} \\
& \times \int_{0}^{L / 2} v(x) \mathrm{d}(x)-C_{p}\left(v^{\prime}(L / 2)-v^{\prime}(0)\right)+H \varphi(0)
\end{aligned}
$$

The boundary conditions for two end clamped (C-C) NWs with a concentrated force $F$ at its mid-point are

$$
\begin{aligned}
v(0) & =0 \\
\varphi(0)= & 0 \\
\varphi(L / 2)= & 0 \\
V(0)= & \alpha_{s} A G\left(v^{\prime}(0)-\varphi(0)\right)-\frac{F}{2}+C_{w} \\
& \times \int_{0}^{L / 2} v(x) \mathrm{d}(x)-C_{p}\left(v^{\prime}(L / 2)-v^{\prime}(0)\right)
\end{aligned}
$$

Due to the symmetry, deflection of the NW for two aforementioned boundary conditions are investigated for $x \in[0, L / 2]$.

The boundary conditions for cantilevered (C-F) NWs can be written as follows

$$
\begin{aligned}
v(0)= & 0 \\
\varphi(0)= & 0 \\
V(0)= & \alpha_{s} A G\left(v^{\prime}(0)-\varphi(0)\right)-F+C_{w} \int_{0}^{L} v(x) \mathrm{d}(x) \\
& -C_{p}\left(v^{\prime}(L)-v^{\prime}(0)\right)-H \varphi(L) \\
-M(0)= & (E I)^{*} \varphi^{\prime}(0)-F L+C_{w} \int_{0}^{L} x v(x) \mathrm{d} x \\
& -C_{p}\left(L v^{\prime}(L)-v(L)\right)-H\left(L \varphi(L)-\int_{0}^{L} \varphi(x) \mathrm{d} x\right)
\end{aligned}
$$




\section{Results and discussion}

\subsection{Effects of size and shear deformation on the normalized contact stiffness of NWs lying on Winkler-Pasternak elastic type substrate}

The contact stiffness which is basically determined under static bending of NW, is defined as

$$
K_{i}=\frac{F_{i}}{v_{i}}
$$

where $F_{i}$ is a force prescribed at point $i$ inducing a deflection $v_{i}$. Here, it is assumed that the point $i$ is the central point for $(\mathrm{C}-\mathrm{C})$ and $(\mathrm{S}-\mathrm{S}) \mathrm{NWs}$ and is at the free end for the (C-F) NWs.

To represent the effects of size (surface) and shear deformation on the contact stiffness of NWs lying on Winkler-Pasternak substrate, we define normalized stiffness of $\bar{K}_{s}$ as below.

$$
\bar{K}_{s}=\frac{K_{s f}}{K_{f}^{E}}
$$

Where $K_{f}^{E}$ denotes the contact stiffness of the conventional E.B.B with two parameters elastic foundation. $K_{s f}$ represents the contact stiffness of a NW modeled by either E.B.B (Euler Bernoulli beam) or T.B (Timoshenko beam) theories lying on the elastic foundation with considering the surface effects.

In other words, $K_{s f}$ is the contact stiffness of a NW with considering both the size-dependence and elastic foundation effects. According to the above definitions, when $K_{s f}$ corresponds to the E.B.B theory, then, $\bar{K}_{s}$ characterizes the attribution of the size-dependency, while, when $K_{s f}$ corresponds to the T.B theory, then, $\bar{K}_{s}$ describes the attribution of both the size-dependency and shear deformation on the stiffness of NWs lying on Winkler-Pasternak elastic substrate.

Variation of the normalized stiffness of a silver NW, $\bar{K}_{s}$, corresponds to the E.B.B and T.B theories versus the aspect ratio $(L / D)$ of the NW are shown in Figure 2 for two diameter values of 20 and $80 \mathrm{~nm}$. The mechanical properties of the silver NW are considered as the following values: $E^{b}=76 \mathrm{GPa}, G=30 \mathrm{GPa}$ and $v=0.26$ where $E^{b}, G$ and $v$ are the Young modulus, shear modulus and Poisson ratio of the bulk of silver NW respectively $[37,51]$. The residual surface stress and the elastic modulus of the surface with crystallographic direction of [001] for a silver NW are: $\tau_{0}^{s}=0.89 \mathrm{~N} . \mathrm{m}^{-1}, E_{s}=1.22 \mathrm{~N} . \mathrm{m}^{-1}$ [10]. The non-dimensional Winkler and Pasternak modulus parameters are considered as $\hat{C}_{w}=30$ and $\hat{C}_{p}=3$ respectively. Figure 2 illustrates the variation of the normalized contact stiffness $\bar{K}_{s}$ and corresponds to a (S-S) NW with respect to the aspect ratio for the two different NW diameters and based on E.B.B and T.B theories. With increasing the aspect ratio $(L / D)$, the NW becomes thinner and the value of surface per volume ratio increases, therefore the effect of surface on the mechanical properties of NW increases. Consequently, the normal stiffness grows in all cases due

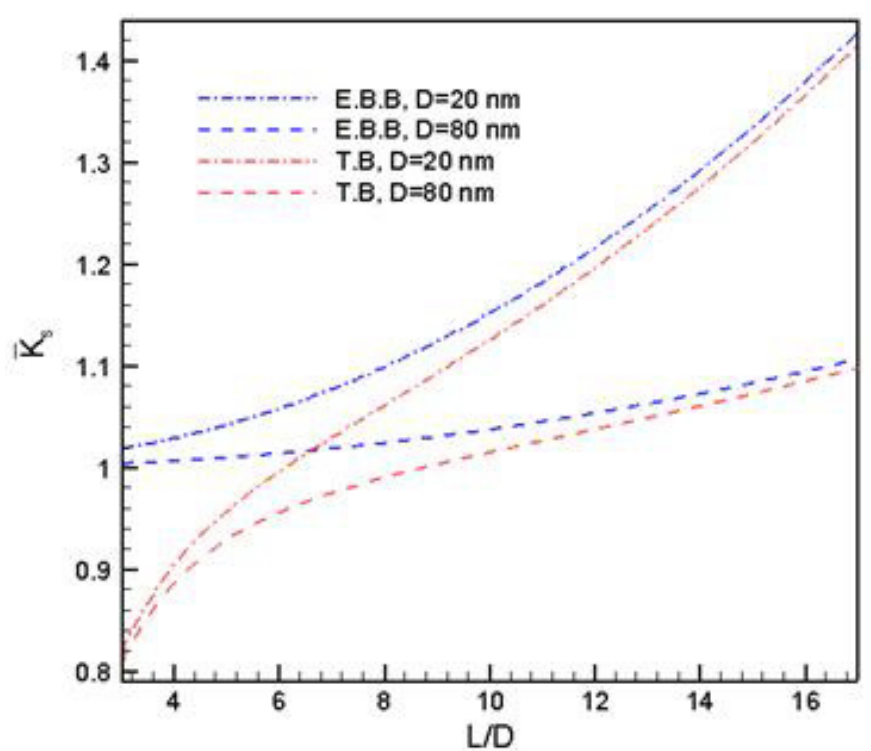

Fig. 2. Normalized contact stiffness of $\bar{K}_{s}$ respect to aspect ratio $(L / D)$ for $(\mathrm{S}-\mathrm{S})$ NWs using by the E.B.B and T.B assumptions.

to the surface effects as shown in Figure 2. This growth is more dominant for the thinner NW $(D=20 \mathrm{~nm})$. Comparing the normal stiffness for NWs with low aspect ratios (small $L / D$ ), obtained by the E.B.B and T.B assumptions in Figure 2, substantial difference is observed. This difference is due to the shear deformation. The shear deformation effect is taken to account in T.B theory while disregarded in E.B.B theory.

By increasing the aspect ratio of NWs, the shear deformation effect decreases and the stiffness of thin NWs resulted by the both theories becomes closed together.

To illustrate the combined effect of size and elastic foundation on the contact stiffness of NWs lying on a substrate, a normal stiffness $\bar{K}_{s f}$ has been defined as

$$
\bar{K}_{s f}=\frac{K_{s f}}{K^{E}}
$$

where $K^{E}$, represents the contact stiffness of the conventional E.B.B contact stiffness of the (without surface effect and elastic foundation) and $K_{s f}$ is defined as before. Variation of the normalized stiffness $\bar{K}_{s f}$ based on E.B.B and T.B theories in terms of aspect ratio $(L / D)$ is shown in Figures $3 \mathrm{a}-\mathrm{c}$ The results are plotted for the three boundary conditions of (S-S), (C-C) and (C-F). For the cases of (S-S) and (C-C) NWs, the normal stiffness $\left(\bar{K}_{s f}\right)$ increases by adding the aspect ratio of NWs; whereas, the normal stiffness for the (C-F) NWs slightly increases at first and, then uniformly decreases. This trend cannot be recognized based on E.B.B theory. By the assumption of E.B.B, the normal stiffness for (C-F) NWs monotonically decreases as the aspect ratio is added.

Again, because of shear deformation, the normal stiffness $\left(\bar{K}_{s f}\right)$ based on T.B theory is smaller than the 


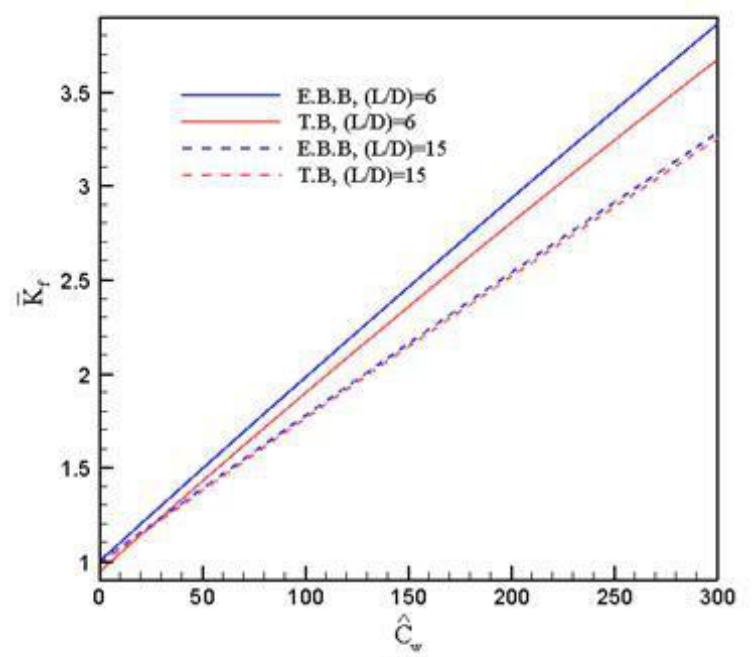

(a)

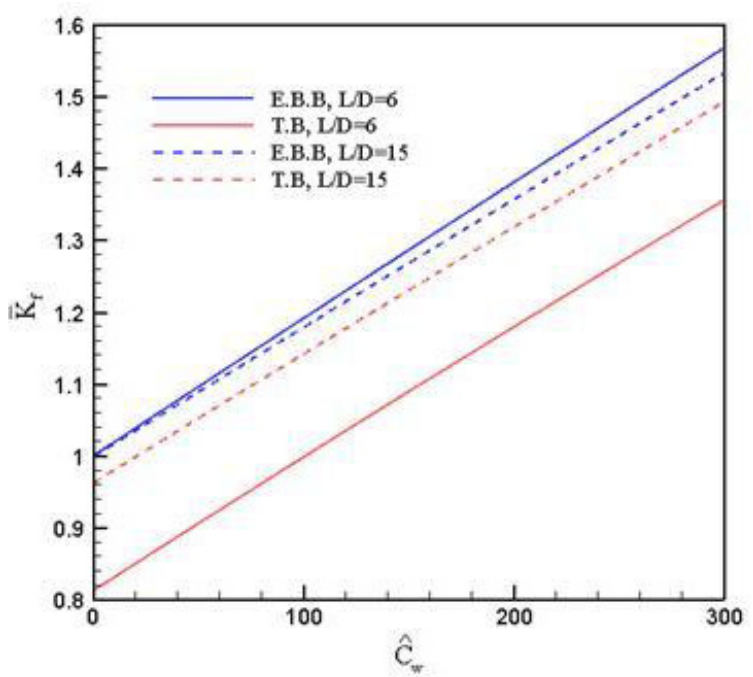

(b)

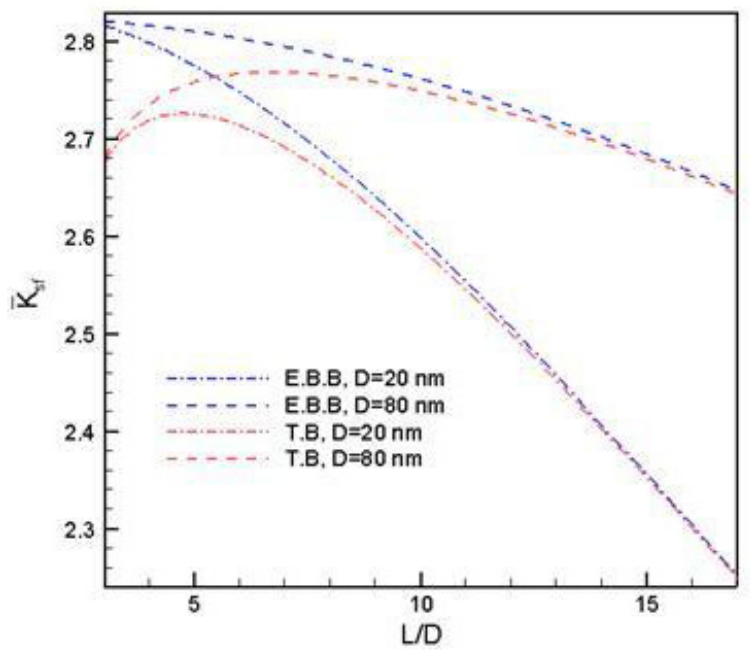

(c)

Fig. 3. Normalized contact stiffness of $\bar{K}_{s f}$ respect to aspect ratio $(L / D)$ obtained by the EBB and TB theories. (a) (S-S) NWs, (b) (C-C) NWs and (c) (C-F) NWs. corresponding normal stiffness based on E.B.B theory for small values of aspect ratios.

It is interesting that for the (C-C) NW (Fig. 3b), the normalized stiffness $\bar{K}_{s f}$ turn out to be unity for a special values of $L / D$. It means that the shear deformation effect overcomes the surface effect and effect of elastic substrate and the NW behaves as simple E.B.B.

\subsection{Effects of elastic foundation and shear deformation on the normalized contact stiffness of NWs lying on Winkler-Pasternak elastic type substrate}

We define normal stiffness of $\bar{K}_{f}$ as follows to describe the foundation effects on the contact stiffness of NWs.

$$
\bar{K}_{f}=\frac{K_{s f}}{K_{s}^{E}}
$$

where, $K_{s}^{E}$ is the contact stiffness of a NW without elastic substrate by considering surface effect based on E.B.B assumption and $K_{s f}$ is defined as before, i.e., $K_{s f}$ is the contact stiffness of a NW with considering both the sizedependency and elastic foundation effects based on either E.B.B or T.B assumption. When $K_{s f}$ corresponds to the E.B.B theory, then, $\bar{K}_{f}$ characterizes the attribution of the elastic substrate, while, as $K_{s f}$ corresponds to the T.B theory, then, $\bar{K}_{f}$ describes the attribution of both the foundation and shear deformation effects on the stiffness of NWs lying on Winkler-Pasternak elastic substrate.

The non-dimensional Winkler and Pasternak modulus parameters for the substrate polymer medium are taken to be in the range of 0 to 300 and 0 to 10 respectively according to Liew et al. [49], since they have used the similar values for modulus of Winkler and Pasternak foundations in vibration analysis of grapheme sheets embedded in polymer matrix. In Figures $4 \mathrm{a}-\mathrm{c}$ and $5 \mathrm{a}-\mathrm{c}$, the influence of the non-dimensional Winkler $\hat{C}_{w}$ and Pasternak $\hat{C}_{p}$ modulus parameters on the local contact stiffness of NWs lying on elastic foundation is investigated using the E.B.B and T.B theories.

\subsubsection{Effect of Winkler modulus parameter $\hat{C}_{w}$ on the normalized contact stiffness of NWs lying on Winkler elastic type foundation $\left(\hat{C}_{p}=0\right)$}

The influence of non-dimensional Winkler modulus parameter $\hat{C}_{w}$ (while $\hat{C}_{p}=0$ ) on the normalized contact stiffness of $\bar{K}_{f}$ has been investigated for two sizes of NWs with geometric dimensions of $L=600 \mathrm{~nm}, D_{1}=40 \mathrm{~nm}$ $(L / D=15)$ and $D_{2}=100 \mathrm{~nm}(L / D=6)$ and the same mechanical properties are considered as the previous example. The results for the three types of boundary conditions of (S-S), (C-C) and (C-F) are shown in Figures $4 \mathrm{a}-\mathrm{c}$. The surface crystallographic direction is [001] for the all cases. The results based on both E.B.B and T.B theories are presented in Figures $4 \mathrm{a}-\mathrm{c}$. As depicted from the figures, the normal stiffness of NWs increases linearly when 


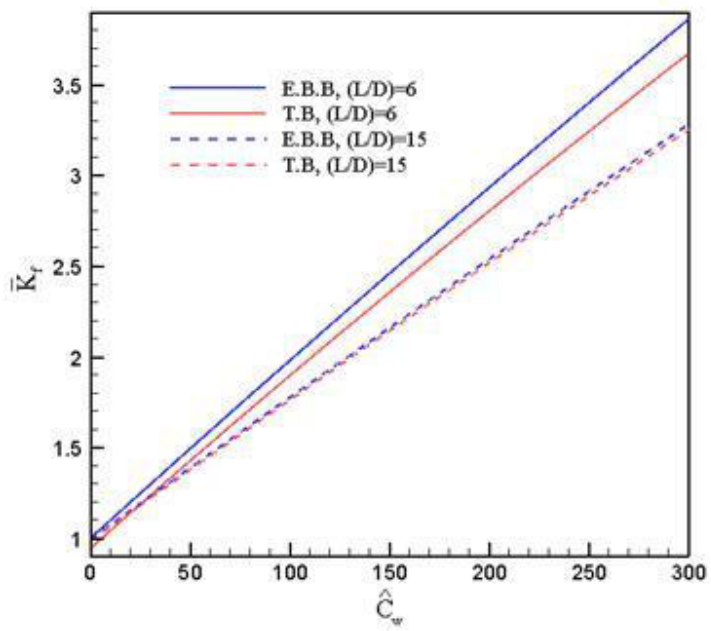

(a)

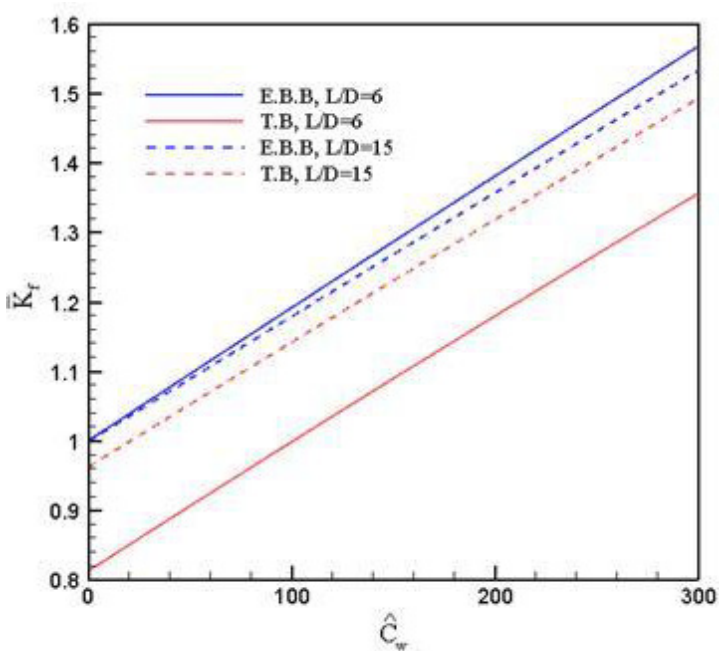

(b)

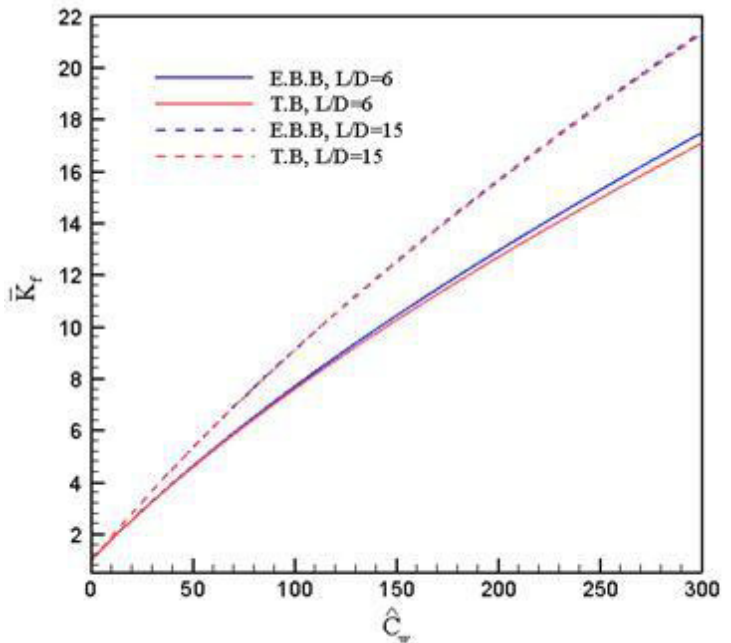

(c)

Fig. 4. EBB and TB solutions of normalized stiffness of $\bar{K}_{f}$ due to increasing the Winkler elastic modulus $\hat{C}_{w}$ of NWs lying on Winkler elastic type substrate $\left(\hat{C}_{p}=0\right)$ and two different aspect ratios, (a) (S-S) NW, (b) (C-C) NW and (c) (C-F) NW.

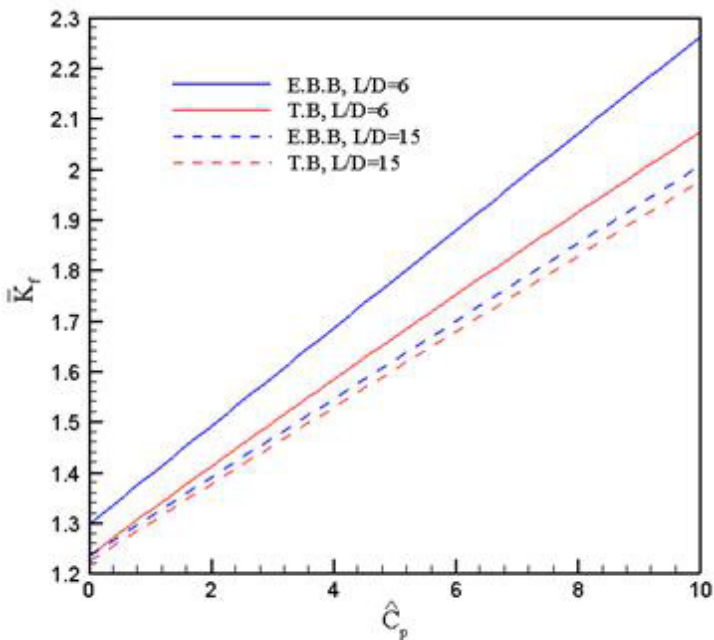

(a)

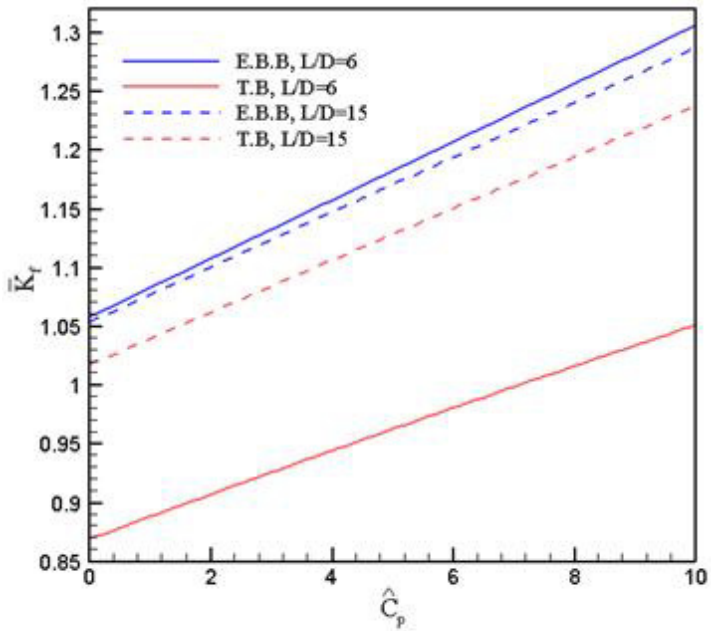

(b)

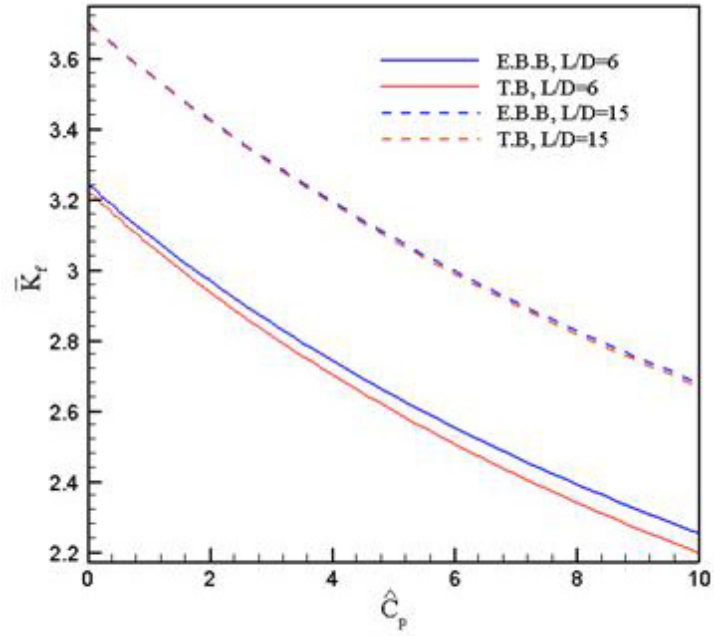

(c)

Fig. 5. EBB and TB solutions of normalized stiffness of $\bar{K}_{f}$ due to increasing the Pasternak elastic modulus $\hat{C}_{p}$ of NWs lying on two parameters Winkler-Pasternak elastic substrate model $\left(\hat{C}_{w}=30\right)$ and two different aspect ratios, (a) (S-S) NW, (b) (C-C) NW, (c) (C-F) NW. 
the Winkler modulus parameter is added. According to the figures, among the three boundary conditions, the (CF) NW is the most affected by the Winkler modulus. It is also depicted from the figures, holding the length of the NW as constant $(600 \mathrm{~nm})$, for the $(\mathrm{S}-\mathrm{S})$ and $(\mathrm{C}-\mathrm{C})$ boundary conditions, the contact stiffness decreases as the aspect ratio $L / D$ increases. While, for the (C-F) NW this trend is reversed. Comparing the results corresponds to the E.B.B and T.B assumptions, again, clear that the shear deformation is more considerable for shorter NWs $(L / D=6)$ and for large values of Winkler parameter.

\subsubsection{Effect of non-dimensional Pasternak modulus parameter $\hat{C}_{p}$ on the normalized contact stiffness of NWs lying on Winkler elastic type foundation}

The effect of non-dimensional Pasternak modulus parameter $\hat{C}_{p}$ on the normalized stiffness $\bar{K}_{f}$ for the silver NWs is investigated in Figures $5 \mathrm{a}-\mathrm{c}$. It is assumed that the Winkler modulus parameter is constant $\left(\hat{C}_{w}=30\right)$. The curves are drawn for two values of aspect ratios $L / D=6$ and $L / D=15$, and the three types of boundary conditions are considered as before. As depicted from the figures, the overall trends for the variations of $\bar{K}_{f}$ in terms of $\hat{C}_{p}$ are the same as $\bar{K}_{f}$ in terms of $\hat{C}_{w}$ for (S-S) and (C-C) NWs. However, for the (C-F) NWs the contact stiffness $\bar{K}_{f}$ decreases as the $\hat{C}_{p}$ is increased. In other words, by increasing the non-dimensional Pasternak modulus parameter $\hat{C}_{p}$ with two values of aspect ratio as $L / D=6$ and $L / D=15$ and the three types of boundary conditions, the (S-S) and (C-C) NWs become stiffer, while, (C-F) NWs become softer.

\subsection{Determining the effective Young's modulus of NWs and size-dependent behavior}

To show the size-dependent mechanical properties of NWs and verifying the presented results, the effective Young's modulus of a clamped-clamped silver NW have been calculated by equating the deflection of the middle points of the present NW and the analytical solution of the classical E.B.B model as follows [36]

$$
\begin{aligned}
\left.v_{N W(c-c)}\right|_{\text {at } x} & =L / 2(\text { from present modeling }) \\
& =\left.\frac{F(3 L-4 x) x^{2}}{48\left(E_{\text {eff }} I\right)}\right|_{\text {at } x=L / 2} \Rightarrow E_{\text {eff }}
\end{aligned}
$$

For determining the effective Young's modulus of a silver NW with the surface crystallographic direction of [001], the parameters are considered as $\tau_{0}^{s}=0.89 \mathrm{~N} . \mathrm{m}^{-1}$, $E_{s}=1.22 \mathrm{~N} . \mathrm{m}^{-1}, E^{b}=76 \mathrm{GPa}, G=30 \mathrm{GPa}, v=0.26$ and $L=800 \mathrm{~nm}$. Figure 6 shows variation of the effective Young's modulus obtained by the above method, versus NW diameter. In this figure, the results obtained by the continuum modeling of NWs based on the E.B.B. and T.B. theories associated with the surface effect are compared with the experimental results of Jing et al. [17].

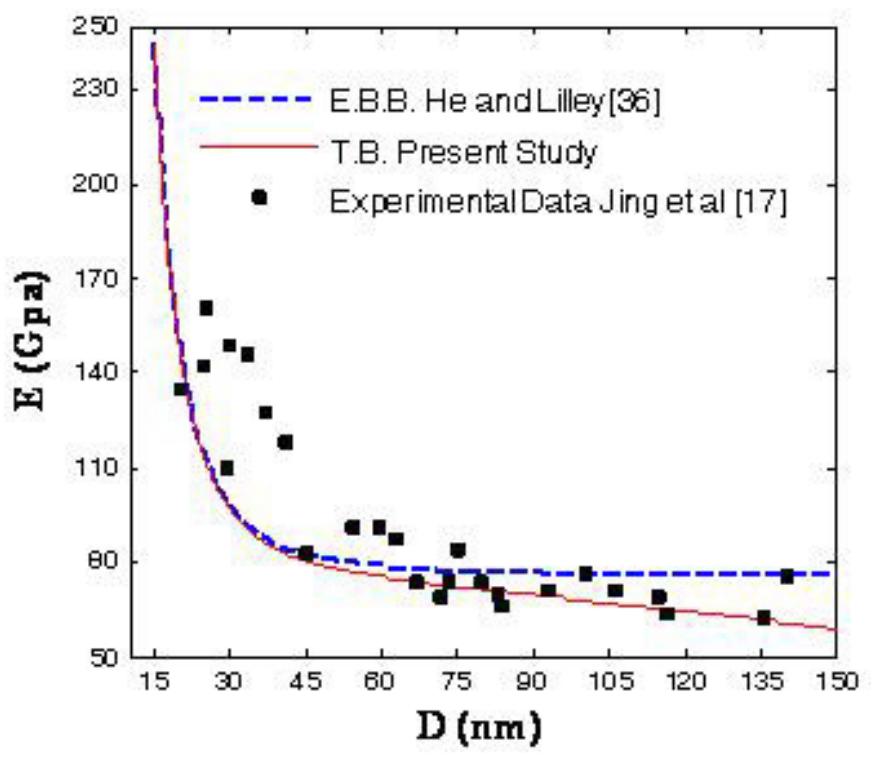

Fig. 6. Effective Young's modulus due to the diameter of silver NW [001] with clamped-clamped boundary condition.

A good agreement between the present approach and the experimental results for a silver NW [001] can be seen in Figure 6. With increasing NW diameter, the effective Young's modulus decreases and when the diameter reaches to a limit value, the size dependency of the Young's modulus is eliminated. For the large values of the aspect ratio $(L / D)$ (thinner NWs), a small alteration in diameter strongly affects the Young's modulus. It is seen in Figure 6 that the T.B theory comparing with the E.B.B theory gives the better prediction for the values of the Young's modulus in the region with the lower values of the aspect ratio. Therefore it is found that for thicker NWs considering the shear deformation is needed.

\section{Conclusion}

In this work, explicit solutions were obtained for static bending response of nanowires (NWs) lying on elastic substrate subjected to a concentrated load. The surface crystallographic direction was considered to be [001]. The influences of different parameters including size, elastic foundation, shear deformation and their combinations were investigated. We used the Timoshenko beam (T.B) theory in tandem with the Laplace-Young equation for the surface effect and Winkler-Pasternak elastic foundation for modeling of the substrate medium. The solutions were obtained for three kinds of boundary conditions: cantilever (C-F), simply supported (S-S) and clamped (C-C). The main findings can be summarized as follows.

1. Based on T.B theory, size-dependency (the effect of surface) of the mechanical properties increases with increasing the aspect ratio of NWs. Accordingly, the rate of variations of stiffness increases when the NWs become thinner. The trend of stiffness variations is different for various boundary conditions. For $(\mathrm{S}-\mathrm{S})$ and 
(C-C) NWs, the contact stiffness of NWs increases by increasing the aspect ratio of NWs, while, the contact stiffness for the (C-F) NWs initially increases slightly, and then decreases uniformly. This behavior for (C-F) NWs cannot be seen based on Euler-Bernoulli beam (E.B.B) theory.

2. For NWs with low aspect ratios (small $L / D$ ), the shear deformation is considerable and cannot be neglected, But for the thinner NWs (large $L / D$ ), the shear deformation effect is negligible. The shear deformation effect is more prominent in the case of (C-C) boundary conditions, and is relatively small for the case of (C-F) boundary conditions.

3. With increasing the Winkler modulus parameter, the contact stiffness of NWs increases approximately as linear for all boundary conditions. Although, the rate of increasing the stiffness is more prominent for (C-F) NWs.

4. With increasing the Pasternak modulus parameter, (S-S) and (C-C) NWs become stiffer, while, (C-F) NWs turn out to be softer.

5. The effect of aspect ratio on stiffness of NWs lying on elastic substrate is more significant in (C-F) type of boundary condition compared to the NWs without foundation.

6. Shear deformation is more considerable for higher values of elastic foundation parameters.

\section{Appendix A. (S-S) NW with complex conjugate roots of characteristic equation}

By defining $p$ and $q$ present in Equations (28a) and (28b) as $p=\Omega / 2$ and $q=\psi / 2$, the constant coefficients $\left(C_{1}-C_{4}\right)$ of Equation (23) are obtained as follows

$$
\begin{aligned}
C_{1} & =\frac{X_{1}}{Y} ; C_{2}=\frac{X_{2}}{Y} ; C_{3}=-C_{1} ; C_{4}=-C_{2} \\
X_{1}= & 4 F \eta\left(\Omega^{2}+\psi^{2}\right)\left\{\Omega\left[4 \alpha \Omega^{2}+\mu-12 \alpha\right]\right. \\
& \times \sin (\psi L) \sinh (\Omega L)+\psi\left[-4 \alpha \psi^{2}+12 \alpha \Omega^{2}+\mu\right] \\
& \times \cos (\psi L) \cosh (\Omega L)\}
\end{aligned}
$$

$$
\begin{aligned}
X_{2}= & -4 F \eta\left(\Omega^{2}+\psi^{2}\right)\left\{\psi\left[4 \alpha \psi^{2}-\mu-12 \alpha \Omega^{2}\right]\right. \\
& \times \sin (\psi L) \sinh (\Omega L)+\Omega\left[-4 \alpha \Omega^{2}+12 \alpha \psi^{2}+\mu\right] \\
& \times \cos (\psi L) \cosh (\Omega L)\}
\end{aligned}
$$

$$
\begin{aligned}
Y=\alpha_{s} A G\left\{\left[A_{8} \psi^{8}+A_{6} \psi^{6}+A_{4} \psi^{4}+A_{2} \psi^{2}+A_{0}\right]\right. \\
\times \sin (\psi L)+\left[A_{5} \psi^{5}+A_{3} \psi^{3}+A_{1} \psi\right] \\
\times \cos (\psi L)\}+C_{w}\left\{\left[B_{4} \psi^{4}+B_{2} \psi^{2}+B_{0}\right] \sin (\psi L)\right. \\
+\left[B_{3} \psi^{3}+B_{1} \psi\right] \cos (\psi L)+\left[\bar{B}_{3} \psi^{3}+\bar{B}_{1} \psi\right] \\
\times \cos ^{2}(\psi L)+\left[\hat{B}_{3} \psi^{3}+\hat{B}_{1} \psi\right]+C_{p}\left\{\left[C_{6} \psi^{6}+C_{4} \psi^{4}+C_{2} \psi^{2}\right.\right. \\
\left.+C_{0}\right] \sin (\psi L)+\left[C_{5} \psi^{5}+C_{3} \psi^{3}+C_{1} \psi\right] \cos (\psi L) \\
\left.+\left[\bar{C}_{5} \psi^{5}+\bar{C}_{3} \psi^{3}+\bar{C}_{1} \psi\right] \cos ^{2}(\psi L)+\left[\hat{C}_{5} \psi^{5}+\hat{C}_{3} \psi^{3}+\hat{C}_{1} \psi\right]\right\} \\
+H\left\{D_{8} \psi^{8}+D_{6} \psi^{6}+D_{4} \psi^{4}+D_{2} \psi^{2}+D_{0}\right\} \sin (\psi L)
\end{aligned}
$$

$$
\begin{aligned}
A_{8}= & -512 \alpha^{2} \sinh (\Omega L) ; \\
A_{6}= & 128 \alpha\left(2 \mu-16 \alpha \Omega^{2}-\eta\right) \sinh (\Omega L) \\
A_{4}= & 32\left(\eta \mu+8 \alpha \Omega^{2} \mu-96\left(\alpha \Omega^{2}\right)^{2}-4 \eta \alpha \Omega^{2}\right) \sinh (\Omega L) \\
A_{2}= & -64 \Omega^{2}\left(4 \alpha \Omega^{2} \mu+\mu^{2}+32\left(\alpha \Omega^{2}\right)^{2}-\eta \mu-2 \eta \alpha \Omega^{2}\right) \\
& \times \sinh (\Omega L) \\
A_{0}= & 32 \Omega^{4}\left(\eta \mu-8 \alpha \Omega^{2} \mu+4 \eta \alpha \Omega^{2}-16\left(\alpha \Omega^{2}\right)^{2}+\mu^{2}\right) \\
& \times \sinh (\Omega L) \\
A_{5}= & 256 \eta \alpha \Omega \cosh (\Omega L) ; A_{3}=512 \eta \Omega^{3} \cosh (\Omega L) ; A_{1} \\
= & 256 \eta \alpha \Omega^{5} \cosh (\Omega L)
\end{aligned}
$$

$$
\begin{aligned}
& B_{4}=-32 \eta \alpha \sinh (\Omega L) ; \\
& B_{2}=\left(192 \eta \alpha \Omega^{2}+4 \eta \mu\right) \sinh (\Omega L) \\
& B_{0}=-\left(32 \eta \alpha \Omega^{4}+\eta \mu \Omega^{2}\right) \sinh (\Omega L) ; \\
& B_{3}=128 \eta \alpha \Omega \cosh (\Omega L) \\
& B_{1}=-16 \eta \Omega\left(8 \alpha \Omega^{2}+\mu\right) \cosh (\Omega L) ; \bar{B}_{3}=-128 \eta \alpha \Omega ; \\
& \bar{B}_{1}=16 \eta\left(8 \alpha \Omega^{3}+\mu \Omega\right) \\
& \hat{B}_{3}=64 \eta \alpha \Omega(1-\cosh (2 \Omega L)) ; \\
& \hat{B}_{1}=8 \eta \Omega\left(\left(\mu+8 \alpha \Omega^{2}\right) \cosh (2 \Omega L)-8 \alpha \Omega^{2}-\mu\right)
\end{aligned}
$$

$C_{6}=-128 \eta \alpha \sinh (\Omega L) ; C_{4}=32 \eta\left(\mu-4 \alpha \Omega^{2}\right) \sinh (\Omega L)$

$C_{2}=64 \eta \Omega^{2}(\mu+2 \alpha) \sinh (\Omega L) ; C_{0}=32 \eta \Omega^{4}\left(\mu+4 \alpha \Omega^{2}\right)$ $\times \sinh (\Omega L)$

$C_{5}=256 \eta \Omega \alpha \cosh (\Omega L) ; C_{3}=512 \eta \Omega^{3} \alpha \cosh (\Omega L)$

$C_{1}=256 \eta \Omega^{5} \alpha \cosh (\Omega L) ; \bar{C}_{5}=-256 \eta \alpha \Omega$;

$\bar{C}_{3}=-512 \alpha \Omega^{3} \eta ; \bar{C}_{1}=256 \alpha \Omega^{5} \eta$

$\hat{C}_{5}=-128 \eta \alpha \Omega(\cosh (2 \Omega L)-1)$;

$\hat{C}_{3}=-256 \eta \alpha \Omega^{3}(\cosh (2 \Omega L)-1)$

$\hat{C}_{1}=-128 \eta \alpha \Omega^{5}(\cosh (2 \Omega L)-1)$ 


$$
\begin{aligned}
& D_{8}=512 \alpha^{2} \sinh (\Omega L) ; D_{6}=256 \alpha\left(8 \alpha \Omega^{2}-\mu\right) \sinh (\Omega L) \\
& D_{4}=32\left(96\left(\alpha \Omega^{2}\right)^{2}+\mu^{2}-8 \alpha \Omega^{2} \mu\right) \sinh (\Omega L) \\
& D_{2}=64 \Omega^{2}\left(\mu^{2}+32\left(\alpha \Omega^{2}\right)^{2}+4 \alpha \Omega^{2} \mu\right) \sinh (\Omega L) \\
& D_{0}=32 \Omega^{4}\left(16\left(\alpha \Omega^{2}\right)^{2}+16 \mu^{2}+8 \alpha \Omega^{2} \mu\right) \sinh (\Omega L)
\end{aligned}
$$

\section{Appendix B. (S-S) NW with real roots of characteristic equation}

The constant coefficients $\left(C_{1}-C_{4}\right)$ of Equation (24) can be written as follows

$$
\begin{gathered}
C_{1}=\frac{X_{11}}{Y_{1}} ; C_{2}=\frac{X_{22}}{Y_{1}} ; C_{3}=-C_{1} ; C_{4}=C_{2} \\
X_{11}=-8 F \eta \Omega \psi^{2}\left(4 \alpha \psi^{2}+\mu\right) \cosh (\psi L) \\
X_{22}=8 F \eta \Omega^{2} \psi\left(4 \alpha \Omega^{2}+\mu\right) \cosh (\Omega L)
\end{gathered}
$$

$$
Y_{1}=\alpha_{s} A G\left[A_{4} \psi^{4}+A_{2} \psi^{2}+A_{0}\right]+C_{w}\left[B_{4} \psi^{4}+B_{2} \psi^{2}+B_{0}\right]
$$$$
+C_{p}\left[C_{4} \psi^{4}+C_{2} \psi^{2}\right]+H\left[D_{4} \psi^{4}+D_{2} \psi^{2}\right]
$$

$$
\begin{gathered}
A_{4}=256 \alpha \Omega^{2}\left[\left(4 \alpha \Omega^{2}+\mu-\eta\right) \cosh (\psi L)\right. \\
\left.-\left(4 \alpha \Omega^{2}+\mu\right) \cosh (\Omega L)\right] \\
A_{2}=64 \Omega^{2}\left[\left(4 \alpha \Omega^{2} \eta+\mu \eta-\mu^{2}-4 \alpha \Omega^{2} \mu\right) \cosh (\Omega L)\right. \\
\left.\quad+\left(4 \alpha \Omega^{2} \mu+\mu^{2}-\mu \eta\right) \cosh (\psi L)\right] \\
\quad \text { B. }
\end{gathered}
$$

$$
\begin{aligned}
B_{4}= & 64 \eta \alpha(1-\cosh (\Omega L)) \cosh (\psi L) \\
B_{2}= & 16 \mu \eta(1-\cosh (\Omega L)) \cosh (\psi L) \\
B_{0}= & 16 \Omega^{2}\left[4 \alpha \Omega^{2} \eta \cosh (\Omega L) \cosh (\psi L)+\eta \mu \cosh (\Omega L)\right. \\
& \left.\times \cosh (\psi L)-4 \eta \alpha \Omega^{2} \cosh (\Omega L)-\mu \eta \cosh (\Omega L)\right]
\end{aligned}
$$

$$
\begin{aligned}
C_{4}= & 256 \alpha \Omega^{2} \eta[(\cosh (\Omega L)-1)] \cosh (\psi L) \\
C_{2}= & 64 \Omega^{2}\left[\left(\eta \mu+4 \alpha \Omega^{2} \eta\right) \cosh (\Omega L)\right. \\
& \left.-4 \alpha \Omega^{2} \eta \cosh (\Omega L) \cosh (\psi L)-\mu \cosh (\psi L)\right] \quad(\mathrm{B} . \\
& \\
D_{4}= & 256 \alpha \Omega^{2}\left[\left(4 \alpha \Omega^{2}+\mu\right)(\cosh (\Omega L)-\cosh (\psi L))\right] \\
D_{2}= & 64 \Omega^{2} \mu\left[\left(4 \alpha \Omega^{2}+\mu\right)(\cosh (\Omega L)-\cosh (\psi L))\right]
\end{aligned}
$$

\section{References}

[1] C.M. Lieber, Nanoscale science and technology: building a big future from small things, MRS Bull 28 (2003) 486491

[2] Y. Xia, P. Yang, Y. Sun, Y. Wu, B. Mayers, B. Gates, Y. Yin, F. Kim, H. Yan, One-dimensional nanostructures: synthesis, characterization, and applications, Adv. Mater. 15 (2003) 353-389

[3] C.M. Lieber, Z.L. Wang, Functional Nanowires, MRS Bull. 32 (2007) 99-108

[4] L.T. Canham, Silicon quantum wire array fabricated by electrochemical and chemical dissolution of wafers, Appl. Phys. Lett. 57 (1990) 1046-48

[5] E.W. Wong, P.E. Sheehan, C.M. Lieber, Nanobeam mechanics: elasticity, strength, and toughness of nanorods and nanotubes, Science 277 (1997) 1971-75

[6] N. Kacem, S. Baguet, S. Hentz, R. Dufour, Nonlinear phenomena in nanomechanical resonators: mechanical behaviors and physical limitations, Mécanique \& Industries 11 (2010) 521-529

[7] Y. Cui, Z. Zhong, D. Wang, W.U. Wang, C.M. Lieber, High performance silicon nanowire field effect transistors, Nano. Lett. 3 (2003) 149-152

[8] M. Cahay, J.P. Leburton, D.J. Lockwood, S. Bondyopadhyay, J.S. Harris, Quantum confinement VI: nanostructured materials and devices, Electrochemical Society, inc. USA, 2001

[9] H. Haug, S.W. Koch, Quantum theory of the optical and electronic properties of semiconductors, World Scientific, Singapore, 2004

[10] V.B. Shenoy, Atomistic calculations of elastic properties of metallic fcc crystal surfaces, Phys. Rev. B. 71 (2005) 094104-11

[11] H.G. Craighead, Nanoelectromechanical systems, Science 290 (2000) 1532-35

[12] K.L. Ekinci, M.L. Roukes, Nanoelectromechanical systems. Rev. Sci. Instrum. 76 (2005) 061101-12

[13] R. Michael, S.C. Wolfram, Handbook of theoretical and computational nanotechnology, American Scientific, 2005, Vol. 1

[14] J. Diao, K. Gall, M.L. Dunn, Atomistic simulation of the structure and elastic properties of gold nanowires, J. Mech. Phys. Sol. 52 (2004) 1935-1962

[15] H.A. Wu, Molecular dynamics study on mechanics of metal nanowire, Mech. Res. Commun. 33 (2006) 9-16

[16] Z.L. Wang, Mechanical properties of nanowires and nanobelts, Dekker Encyclopedia of Nanoscience and Nanotechnology (2004) 1773-1786, DOI: $10.1081 / E-E N N .120013387$

[17] G.Y. Jing, H.L. Duan, X.M. Sun, Z.S. Zhang, J. Xu, Y.D. Li, J.X. Wang, D.P. Yu, Surface effects on elastic properties of silver nanowires: Contact atomic-force microscopy, Phys. Rev. B 73 (2006) 235409-6

[18] Y.X. Chen, B.L. Dorgan, D.N. McIlroy, D.E. Aston, On the importance of boundary conditions on nanomechanical bending behavior and elastic modulus determination of silver nanowires, J. Appl. Phys. 100 (2006) 104301-7

[19] C.Q. Chen, Y.S. Zhang, J. Zhu, Y.J. Yan, Size dependence of Young's modulus in $\mathrm{ZnO}$ nanowires, Phys. Rev. Lett. 96 (2006) 075505-4

[20] R.E. Miller, V.B. Shenoy, Size-dependent elastic properties of nanosized structural elements, Nanotechnology 11 (2000) 139-147 
[21] J.-G. Guo, Y.-P. Zhao, The size-dependent bending elastic properties of nanobeams with surface effects, Nanotechnology 18 (2007) 295701-6

[22] X.F. Li, B.L. Wang, K.Y. Lee, Size effects of the bending stiffness of nanowires, J. Appl. Phys. 105 (2009) 074306-6

[23] M.E. Gurtin, A.I. Murdoch, A continuum theory of elastic material surfaces, Arch. Rational. Mech. Anal. 57 (1975) 291-323

[24] R. Dingreville, J. Qu, M. Cherkaoui, Surface free energy and its effect on the elastic behavior of nano-sized particles, wires and films, J. Mech. Phys. Solids 53 (2005) $1827-1854$

[25] H.L. Duan, J. Wang, Z.P. Huang, B.L. Karihaloo, Eshelby formalism for nano-inhomogeneities, Proc. R. Soc. A 461 (2005) 3335-3353

[26] H.L. Duan, J. Wang, Z.P. Huang, B.L. Karihaloo, Sizedependent effective elastic constants of solids containing nano-inhomogeneities with interface stress, J. Mech. Phys. Solids 53 (2005) 1574-1596

[27] H.L. Duan, X. Yi, Z.P. Huang, J. Wang, A unified scheme for prediction of effective moduli of multiphase composites with interface effects, Part I: Theoretical framework, Mech. Mater. 39 (2007) 81-93

[28] P. Sharma, S. Ganti, N. Bhate, Effect of surfaces on the size-dependent elastic state of nano-inhomogeneities, Appl. Phys. Lett. 82 (2003) 535-537

[29] P. Sharma, S. Ganti, Size-dependent Eshelby's tensor for embedded nano-inclusions incorporating surface/interface energies, ASME J. Appl. Mech. 71 (2004) $663-71$

[30] J. Yvonnet, H.L. Quang, Q.-C. He, An XFEM/level set approach to modelling surface/interface effects and to computing the size-dependent effective properties of nanocomposites, Comput. Mech. 42 (2008) 119-131

[31] J. Yvonnet, A. Mitrushchenkov, G. Chambaud, Q.-C. He, Finite element model of ionic nanowires with sizedependent mechanical properties determined by ab initio calculations, Comput. Methods. Appl. Mech. Eng. 200 (2011) 614-625

[32] G.F. Wang, X.Q. Feng, Effects of surface elasticity and residual surface tension on the natural frequency of microbeams, Appl. Phys. Lett. 90 (2007) 231904

[33] G.F. Wang, X.Q. Feng, Effects of surface stresses on contact problems at nanoscale, J. Appl. Phys. 101 (2007) 013510-6

[34] G.F. Wang, X.Q. Feng, Surface effects on buckling of nanowires under uniaxial compression, Appl. Phys. Lett. 94 (2009) 141913

[35] G.F. Wang, X.Q. Feng, Timoshenko beam model for buckling and vibration of nanowires with surface effects, J. Phys. 42 (2009) 155411
[36] J. He, C.M. Lilley, Surface effect on the elastic behavior of static bending nanowires, Nano Lett. 8 (2008) 1798-1802

[37] L.Y. Jiang, Z. Yan, Timoshenko beam model for static bending of nanowires with surface effects, Physica E: Low-dimens. Syst. Nanostruct. 42 (2010) 2274

[38] M.C.P. Wang, B.D. Gates, Directed assembly of nanowires, Mater. Today 12 (2009) 34-43

[39] W. Lu, C.M. Lieber, Nanoelectronics from the bottom up, Nat. Mater. 6 (2007) 541-850

[40] C.Q. Ru, Axially compressed buckling of a doublewalled carbon nanotube embedded in an elastic medium, J. Mech. Phys. Solids 49 (2001) 1265-79

[41] S.C. Pradhan, A. Kumar, Vibration analysis of orthotropic graphene sheets embedded in Pasternak elastic medium using nonlocal elasticity theory and differential quadrature method, Comput. Mater. Sci. 50 (2010) 239245

[42] M. Mohammadimehr, A.R. Saidi, A. Ghorbanpour Arani, A. Arefmanesh, Q. Han, Torsional buckling of a DWCNT embedded on Winkler and Pasternak foundations using nonlocal theory, J. Mech. Sci. Technol. 24 (2010) 12891299

[43] R.F. Scott, Foundation Analysis, Prentice-Hall: Englewood Cliffs N.J, 1981

[44] A.C. Ugural, S.K. Fenster, Advanced strength and applied elasticity, Printice Hall: USA, 2003

[45] P.L. Pasternak, On a new method of analysis of an elastic foundation by means of two foundation constants, Goz Izd Lip Po Strait i Arkh: Moscow (in Russian), 1954

[46] R.C. Cammarata, Surface and interface stress effects in thin films, Prog. Surf. Sci. 46 (1994) 1-38

[47] J.R. Hutchinson, Shear coefficients for timoshenko beam theory, J. Appl. Mech. Trans: ASME 68 (2001) 87-92

[48] A. Ghani razaqpur, K.R. Shah, Exact analysis of beams on two-parameter elastic foundations, Int. J. Solids Struct. 27 (1991) 435-54

[49] K.M. Liew, X.Q. He, S. Kitipornchai, Predicting nanovibration of multi-layered graphene sheets embedded in an elastic matrix, Acta. Mater. 54 (2006) 4229-4236

[50] T. Murmu, S.C. Pradhan, Buckling analysis of a singlewalled carbon nanotube embedded in an elastic medium based on nonlocal elasticity and Timoshenko beam theory and using DQM, Physica E: Low-dimens. Syst. Nanostruct. 41 (2009) 1232-1239

[51] X.F. Li, G.T. Fei, W.F. Zhou, L.D. Zhang, A convenient method to determine the bulk modulus of nanowires and its temperature dependence based on X-ray diffraction measurement, Solid. State. Commun. 150 (2010) 11171119 